\title{
MUSIC THERAPY IN RETT SYNDROME CASES
}

\author{
Vita Andziule \\ Special Kindergarten "Ciauskutis”, Lithuania \\ Vilmante Aleksiene \\ Lithuanian Academy of Music and Theatre, Lithuania \\ Sigita Lesinskiene \\ Vilnius University Faculty of Medicine, Lithuania
}

\begin{abstract}
Rett syndrome is a rare (0,01\%) genetic disorder affecting girls' development. Individuals with Rett syndrome experience a full range of health problems that severely influence and complicate their mobility, intellect, communication, learning, and entire lifestyle. The treatment of Rett's syndrome is still aimed at investigating new pathways to ensure the best possible patient's development and quality of life. The distinctive feature of Rett syndrome is the two main sensations with little damage: hearing and vision. Therefore, music therapy plays an exceptional role in the treatment and special education of children with Rett syndrome. The goal of this study is to find the most effective music therapy techniques and their specific indications whilst working with individuals with Rett syndrome. Research method used: music therapists' online survey conducted internationally. The research suggests that the most effective methods in Rett syndrome cases are structured musical games that promote targeted hands-on movements, as well as listening to the songs and choice of favourite songs using pictures or other symbols. Music therapy can be applied for various health needs of individuals with Rett syndrome, especially to enhance hand functions as well as to improve non-verbal communication and to help individuals experience togetherness and joy.
\end{abstract}

Keywords: Rett syndrome, music therapy methods / techniques.

\section{Introduction}

Rett syndrome is a rare $(0.01 \%)$ girl-specific genetic developmental disorder severely affecting physical and mental abilities. At the time when this disorder was described for the first time by Andreas Rett in 1966 (as cited in Chahrour \& Zoghbi, 2007: 422) gene mutations causing it were not yet known, and therefore, based on similar clinical symptoms such as limited eye contact and poor social interaction, Rett syndrome was classified as one of the autism spectrum disorders (DSM-3). Recent studies have revealed that Rett syndrome has genetic origin mutations in the gene MECP2 which is located on the $\mathrm{X}$ chromosome and is responsible for the production of protein necessary to maintain normal brain functions are found in $95 \%$ of cases (Chahrour \& Zoghbi, 2007; Percy, 2011; Bhandari, 2015). 
Diagnostic criteria for Rett syndrome have also been revised (Perry, 1991; Hunter, 2007; Scruggs, 2009; Percy 2011; Lotan \& Merrick, 2011). Overlapping features have been identified in the variety of scientific findings, first of all, the fact that psychomotor developmental disorders become apparent after normal prenatal and perinatal periods, between 6 and 18 months of age (Scruggs, 2009). Secondly, girls who have this disorder grow slower than other children in their early childhood, they often have a smaller head (microcephaly), their abilities to move (walk) and purposefully use their hands regress, and stereotypic hand movements such as clapping, wringing, tapping occur instead. Other emerging symptoms include disorders in cognitive functions and speech, sensory system disorders (except for vision and hearing), respiratory abnormalities (periods of apnoea or hyperventilation during wakefulness), seizures, scoliosis, sleep disorders (Hunter, 2007; Lotan \& Merrick, 2011; Percy, 2011).

Studies show that the survival of girls with this disorder until the adult age although accompanied by sickliness, complications, loss of motor, cognitive, social abilities - is probable (Hunter, 2007). It has also been proven that girls with Rett syndrome are capable of learning new skills, provided that appropriate conditions are created for their education (Wesecky, 1986; Merker, BergstroemIsacsson, \& Engerstroem 2001; Elefant \& Wigram, 2005; Hunter, 2007). These recent data change the understanding of Rett syndrome and challenge professionals. Along with regular medical studies conducted around the world and aimed at controlling mutations in the gene causing Rett syndrome, there is a strong need for studies on ways to control and/or reduce symptoms. The strategy for helping people with Rett syndrome, aimed at applying effective forms and methods of therapy, nursing and education, is still being developed.

Rett syndrome cannot be cured, and some symptoms of the syndrome (e.g. epileptic seizures, severe respiratory, sleep disorders, osteoporosis) can only be controlled by medication. However, the disorder is complex, with each case being unique, therefore it requires interdisciplinary client-oriented work, while complementing therapeutic medical interventions with alternative therapies, focusing on specific abilities and strengths of an individual - especially on the least affected hearing and vision functions. Patients are usually recommended kinesitherapy, massages, occupational therapy, music therapy, hydrotherapy and hippotherapy. Neither of them can be perceived as the main one, but at the same time each one is both important and complementary, aimed at improving the functionality of individuals with Rett syndrome, their ability to communicate, and move (Hunter, 2007; Didden et al., 2010; Lotan \& Merrick, 2011).

Music therapy nonetheless has an exclusive role in the list of therapies for individuals with Rett syndrome. It was recommended by Andreas Rett himself as a useful intervention for reducing stereotypic hand movements, shortening response time, muscle tension, arousing interest and stimulating the sensory 
system. Girls with Rett syndrome are sensitive, receptive to musical sounds, able to recognise and associate them with events, so music therapy can be particularly successful in promoting and motivating communication with the environment (Lotan \& Elefant, 2004). Individuals with Rett syndrome are particularly fond of music, therefore, by using music as their strong side, one can contribute to smoother development and education of these patients because learning new things is easier when the learning content is presented in an interesting way, and the process is engaging (Hunter, 2007). Andreas Rett noticed that music could break the barrier of difficulties, and at the same time revealed the hidden capabilities of patients with Rett syndrome (Chahrour \& Zoghbi, 2007). Parents, therapists, physicians, in describing girls with Rett syndrome, mention that their connection with people and the world is created through music, and it is music that helps them understand and interpret themselves in the first place, as well as their surrounding environment and relationships with people (Merker et al., 2001; Trevarthen \& Burford, 2001). In addition, it is very important that girls with Rett syndrome have an experience of normal development in the first year of their life until about 18 months. It should be noted that there is an intact period of early musical communication (Trevarthen \& Malloch, 2000), and this is one of the strongest internal resources. A music therapist can use this form of musical interaction which is known to a child from the early days and gives a child a feeling of safety to stimulate the development of general abilities of later stages.

However, experience of applying music therapy with this rare group of patients is still severely under-studied, and there has been only patchy analysis of the music therapy technique for work with individuals who have Rett syndrome.

Research questions: What methods / techniques do music therapists use on their practice with Rett syndrome clients?

The goal is to reveal the most effective music therapy techniques and their specific indications whilst working with individuals with Rett syndrome.

The hypothesis is that music therapy improvisation techniques are the most suitable for reducing stereotypic hand movements and increasing targeted movements.

\section{Research methodology}

The combined quantitative - qualitative study was carried out. Research methods: 1) music therapists' online survey conducted internationally; 2) video microanalysis and descriptive case study. In this article we present only a quantitative part of the study.

Music therapists' online questionnaire-based survey "Music Therapy for the Development of Individuals with Rett Syndrome: Interventions, Methods in Practice" was conducted in the first stage of the research (January - April 2017). 
The survey was meant for foreign music therapists, since there were no music therapists in Lithuania working on Rett syndrome cases at that time. The questionnaire was originally developed and the questions were based on studies of literature and on theoretical assumptions. Requests to complete the questionnaire were sent to music therapy centres in 27 countries, and to Rett syndrome centres in the United States (Texas, California), Sweden, Austria, and Spain. The questionnaire was submitted and the statistical data were processed using qualtrics.com software. The data analysis was performed in May 2017.

\section{Questionnaire survey results}

A professional music therapist either working or having worked with the Rett syndrome cases is the key criterion enabling participation in the survey. All in all 38 responses have been received with just 11 of them meeting the stipulated criterion. Demographic distribution of respondents is as follows: 8 individuals from the USA, 1 from Sweden and Austria, while receipt of 1 survey reply has been failed.

The age of respondents having participated in the survey varied from 24 to 59 years old and work experience as music therapists has also been versatile enough, ranging from less than 1 year to even 37 years.

Non-representativeness of the survey sample prevents making a more general summarisation. Nevertheless, considering the rarity of Rett syndrome, the survey results reveal general tendencies, when music therapy is applied to individuals with Rett syndrome.

The immediate attempt has been made to clarify the type and frequency of music therapy methods applied or recommended by the professionals in order respective heath care needs of girls with Rett syndrome are met. The derived data are presented in Figure 1.

The most applicable and recommendable music therapy methods are playing the songs which are familiar to patients and alternating them with pauses intended for the patients to respond (to press a button in order to complete a musical phrase etc.) and the very moment of choosing the songs (by a head tilt or finger movement), and playing musical games with instruments when their direction is changed or a single instrument is shared. The ability of individuals with Rett syndrome to choose by demonstrating their musical predilections or preferences was described by C. Elefant, music therapist. In her dissertation she has communicated an important message about the ability of the patients to express their wishes and emotions, since these appear to be among the critical factors of the quality of life (Elefant, 2002). Music therapy methods applied the least include improvisations directed towards synchronization with the child's movements or breathing, or directive teaching tasks (timely inhaling, fulfilling a task, etc.), or 
abrupt dynamic changes. Notwithstanding positive response to music of the patients suffering from dysfunctional breathing or great communication and social disorders, it may evoke overstimulation, hyperventilation or overreaction (Wesecky, 1986; Merker, et al., 2001). Moreover, vibroacoustic music therapy, as another effective music therapy method, has been discovered. This method as being effective for reduction of the risk caused by breathing anomalies has been indicated by even 2 respondents.

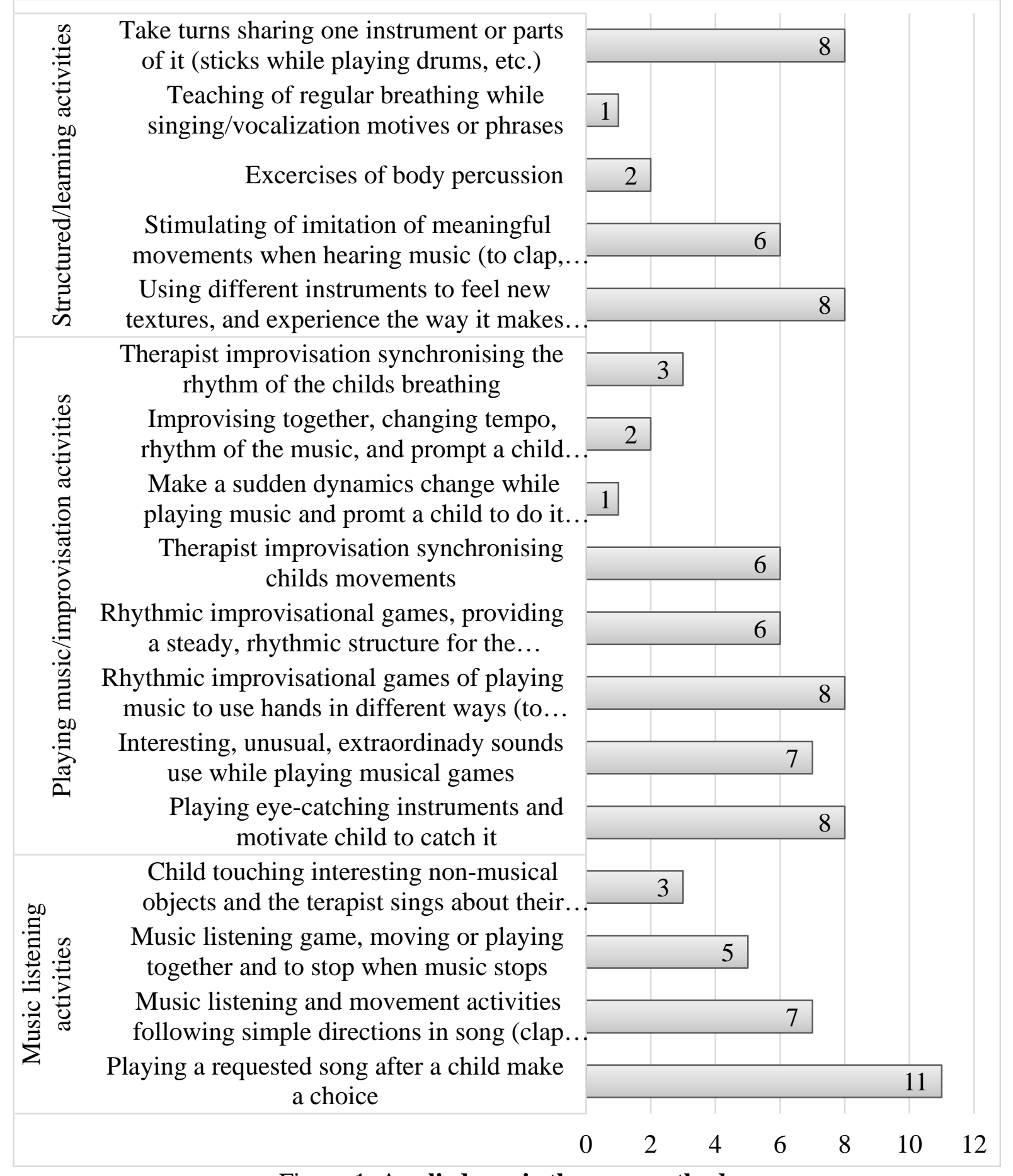

Figure 1. Applied music therapy methods 
The respondents have been asked to assess the degree of applicability of music therapy to meet different health care needs of individuals with Rett syndrome, where replies 'definitely non-recommendable' accounted for 1 score, 'rather no than yes' - 2, 'rather yes than no' - 3, and 'highly recommendable' 4. Thus, 44 being the biggest possible sum of the collected scores. The results are illustrated in Figure 2.

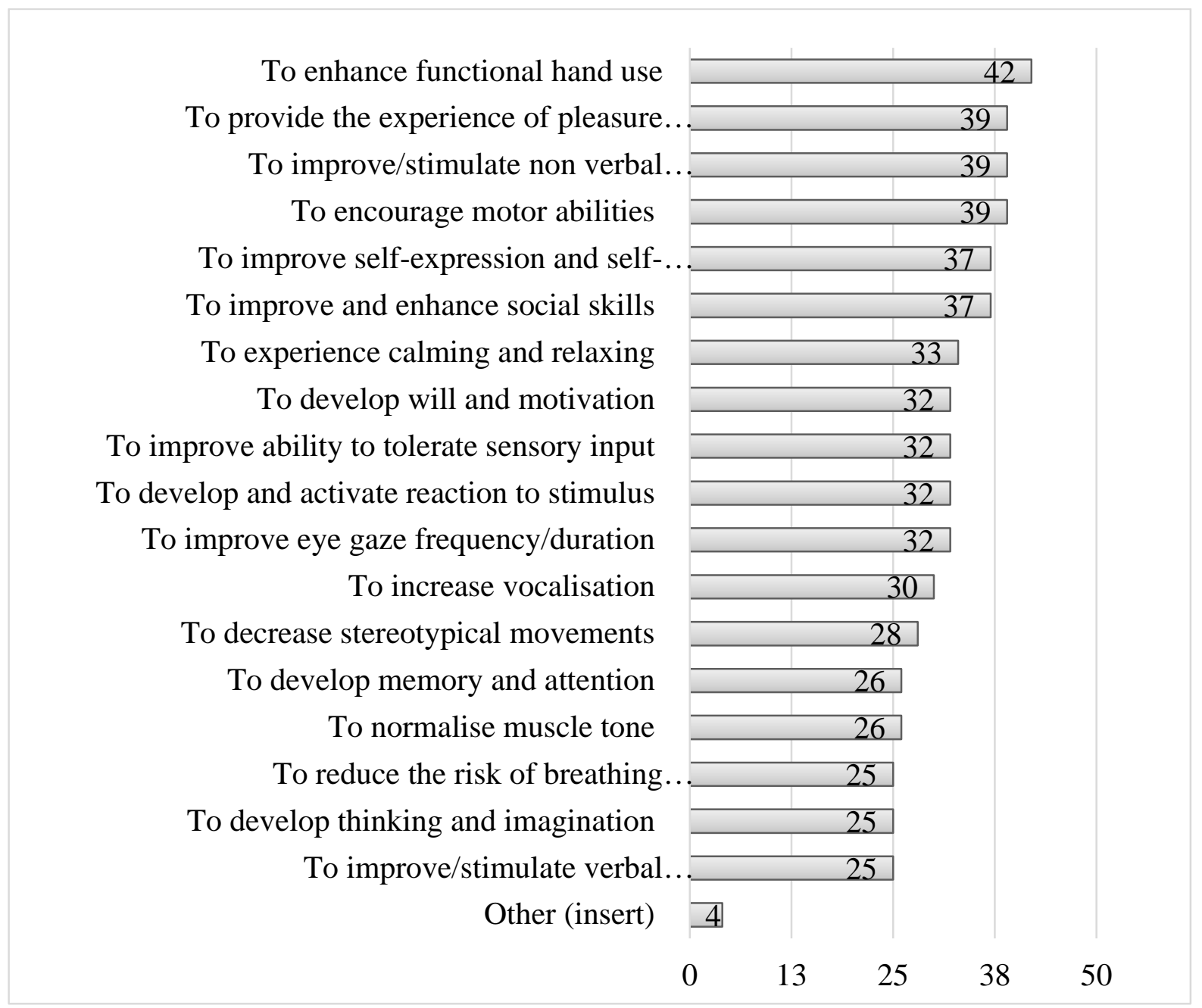

Figure 2. Indications for music therapy

To the opinion of the questionnaire survey respondents, music therapy is the most indicated for the development of functional use of hands for girls with Rett syndrome (42). Interventions of music therapy indicated for other health care needs include: maintaining or strengthening of the body motor or movement functions (39), improvement of non-verbal communication (39), experiencing joy and pleasure (39). Also, though to a slightly smaller extent, this kind of therapy is recommendable for the improvement of integration of self-concept and selfexpression (37), social skills (37) and tactile sensations (36). Thus, music therapy 
is applied both as the medical intervention and the key motivator and mediator assisting in communication with patients with Rett syndrome. Versatile music therapy methods are applied, however, priority of the interviewed music therapists has been given to listening of the chosen songs and the very moment of choosing the songs, as well as the structured musical games relying on creative use of different timbre and texture instruments.

It has been revealed by our study that music therapy interventions are less successful, i.e. less recommendable for the development of verbal communication of children with Rett syndrome (25). The results of this study are supported by previous studies which indicate that female patients with Rett syndrome mostly communicate and express themselves by mimics and body language with just negligible vocalisations. Their vocal signals are hard to grasp and read due to excessively large or small tone of their facial and body muscles (Lotan \& Elefant, 2004; Hunter, 2007). Again, smaller potential of music therapy is reported for the development of thinking and imagination (25), memory and attention (26), and normalisation of muscles tone (26), and minimisation of risks caused by breathing abnormalities (25). Music therapists select and try music therapy intervention methods in an exceptionally responsible manner. Primarily, creation of the surroundings acceptable and comprehensible to the patient is ensured aiming at successful participation of the patient. Strengthening of a positive attitude of the patient is attempted in order to enable the development of physical, emotional and cognitive capacities of the patient (Yasuhara \& Sugiyama, 2001; Lotan \& Elefant, 2004; Wigram \& Lawrence, 2005).

Varied frequency of sessions involving music therapy for the patients with Rett syndrome is reported in the practice of music therapists having participated in the survey: 6 respondents conduct music therapy sessions 1 time a week, 4 respondents have such sessions 2-3 times a week and a reply of 1 respondent reads 'in periods 3-5 per week'. The most popular duration of the music therapy session is 30 minutes - such a reply has been chosen by 7 respondents.

Also, the first improvement signals have been noticed in the course of the first weeks of therapy by 7 respondents while other respondents have indicated a slightly later time span, but not later than half a year. Besides a reply given by 1 respondent about noticing an improvement read 'almost at once'. These results suggest about the positive effect of music therapy.

\section{Conclusions}

1. The results of internationally-conducted questionnaire survey indicate that the most often applied the most effective music therapy methods for the patients with Rett syndrome include: structured musical games using musical instruments of interesting texture, different timbre and varying touch pattern; 
selection of songs and instrumental music pieces using pictures or other symbols; listening to songs or short instrumental pieces and motivating a child to purposefully fill in a pause with a musical instrument or to clap hands when a basic beat is heard, or the like. These music therapy methods are indicative aiming at functional use of hands and strengthening of other motor skills; also at improvement of non-verbal communication and experiencing joy and pleasure.

2. The music therapists' survey did not prove the hypothesis on the applicability/suitability of improvisational music therapy methods for the reduction of stereotypic hand movements. Besides, music therapy if applied for the purposes of development of verbal communication, thinking and imagination, and for normalisation of muscles tone or minimisation of breathing abnormalities is considered ineffective. However proof about its non-applicability/unsuitability is insufficient due to non-representativeness of the survey sample and a particularly small degree of the problem investigation in scientific literature.

\section{Discussion}

Along with continuous researches pursued on the global scope, music therapy researches are necessary indeed to control and/or minimise manifestation of symptoms with due attention drawn to distinctive abilities and strengths and to the least adversely affected hearing and vision functions in particular of the individuals with Rett syndrome. The main tasks for the girls are to learn nonverbal communication, express their wishes and emotions, whereas individuals who surround the patients need learning how to understand them and motivate their interaction relying on different communication means, say, communication boards, pictures or other symbols. In general, communication is considered to be the fundamental demand of humans and the lack of it leads to dissatisfaction, frustration and social segregation, that is why it is of utmost importance that the emphasis is made on learning to express ones wishes and emotions in interaction with others, as the ability to communicate alone is not enough. For this purpose the music communication which is particularly suitable for and mostly liked by the individuals with Rett syndrome is available. Furthermore, it enables them to keep connection with another person and to be understood.

The questionnaire survey results reveal the specifics and complexity of the application of music therapy for the Rett syndrome cases. The specific applicability of music therapy for the individuals with Rett syndrome proves the emergence of the first communication improvement signals already in the course of the first sessions. 
Development of technologies introduces new means of therapy, e.g. Vibroacoustic therapy, and communication, e.g. Assistive Augmentative Communication. The latter tool enables computer management by the patients with just a single movement of a finger, a released sound or even a glance. Music communication technologies are also taking a rapid pace of development and their successful application in the processes of therapy and teaching of the Rett syndrome patients is highly probable in the future.

\section{References}

Bhandari, S. (2015). Rett Syndrome Symptoms, Causes, and Treatments. Retrieved from http://www.webmd.com/brain/autism/rett-syndrome?page=2\#1

Chahrour, M., \& Zoghbi, Y. H. (2007). The Story of Rett Syndrome: From Clinic to Neurobiology. Neuron, 56, 422-437. Retrieved from http://dx.doi.org/10.1016/ j.neuron.2007.10.001

Didden, R., Korzilius, H., Smeets, E., Green, V. A., Lang, R., Lancioni, G. E., \& Curfs, L. M. (2010). Communication in Individuals with Rett Syndrome: an Assessment of Forms and Functions. Journal of Developmental and Physical Disabilities, 22 (2), 105-118. Retrieved from http://doi.org/10.1007/s10882-009-9168-2

Elefant, C. (2002). Enhancing Communication in Girls with Rett Syndrome through Songs in Music Therapy. Aalborg: Aalborg Universitet. Retrieved from vbn.aau.dk

Elefant, C., \& Lotan, M. (2004). Rett syndrome: Dual intervention music and physical therapy. Nordic Journal of Music therapy, 13 (2), 172-182.

Elefant, C., \& Wigram, T. (2005). Learning ability in children with Rett syndrome. Brain and Development, 27 (1), 97-101.

Hunter, K. (2007). The Rett Syndrome Handbook. Clinton, MD: International Rett Syndrome Foundation Publishing.

Yasuhara, A., \& Sugiyama, Y. (2001). Music therapy for children with Rett syndrome. Retrieved from http://dx.doi.org/10.1016/S0387-7604(01)00336-9

Lotan, M., \& Merrick, J. (2011). Rett Syndrome: Therapeutic Interventions. New York: Nova Science Publishers.

Merker, B., Bergstroem-Isacsson, M., \& Engerstroem, I. W. (2001). Music and the Rett Disorder: The Swedish Rett Center Survey. Nordic Journal of Music Therapy, 10 (1), 45-53.

Percy, A. K. (2011). Rett Syndrome: Exploring the Autism Link. Archives of Neurology, 68 (8), 985-989. Retrieved from http://doi.org/10.1001/archneurol.2011.149

Perry, A. (1991). Rett syndrome: A comprehensive review of the literature. American Journal on Mental Retardation, 96, 275-290.

Scruggs, A. (2009). Rett Syndrome: Characteristics, Causes, and Treatment. Thesis, Lynchburg College, 2009. Retrieved from http://www.lynchburg.edu/wp-content/ uploads/volume-4-2009/ScruggsA-Rett-Syndrome-Characteristics-CausesTreatment.pdf

Trevarthen, C., \& Burford, B. (2001). Early infant intelligence and Rett syndrome. In A. Kerr \& I. Witt Engerstrom (Eds.), Rett disorder and the developing brain, 303-326. New York: Oxford University Press 
Andziule et al., 2018. Music Therapy in Rett Syndrome Cases

Trevarthen, C., \& Malloch, S. (2000). The Dance of Wellbeing: Defining the Music Therapeutic Effect. Nordic Journal of Music Therapy, 9 (2), 3 - 18.

Wesecky, A. (1986). Music therapy for children with Rett Syndrome. American Journal of Medical Genetics, 24 (1), 253-257.

Wigram, T., \& Lawrence, M. (2005). Music therapy as a tool for assessing hand use and communicativeness in children with Rett syndrome. Brain \& Development, 27, 95-96. 